\title{
Optimizing Two-Photon Initiators and Exposure Conditions for Three-Dimensional Lithographic Microfabrication
}

\author{
Stephen M. Kuebler, Mariacristina Rumi, Toshiyuki Watanabe, ${ }^{a}$ Kevin Braun, \\ Brian H. Cumpston, Ahmed A. Heikal, Lael L. Erskine, S. Thayumanavan, Stephen Barlow, \\ Seth R. Marder*, and Joseph W. Perry* \\ Department of Chemistry, the University of Arizona, Tucson, AZ 85721 \\ ${ }^{a)}$ Permanent address: Department of Applied Chemistry, Faculty of Technology, Tokyo University of \\ Agriculture \& Technology, 2-24-16 Nakamachi, Koganei-shi, Tokyo 184-8588, Japan \\ *to whom correspondence should be addressed.E-mail for JWP: jwperry@u.arizona.edu; \\ e-mail for SRM: smarder@u.arizona.edu.
}

\begin{abstract}
The radical photoinitiation characteristics of a range of $D-\pi-D$ chromophores (where $D$ is an electron-donor and $\pi$ is a biphenyl, stilbene, or bis(styryl)benzene conjugated bridge) under two-photon excitation are reported. Photo-crosslinkable resins were formulated with these initiators and were used to fabricate a variety of complex three-dimensional structures by two-photon induced polymerization (TPIP). These structures illustrate that TPIP can be a highly versatile technique for the rapid single-step fabrication of complex microstructures and devices. The new two-photon resins were found to be as much as 50 times more sensitive than resins containing conventional UV initiators. The increased two-photon sensitivity is attributed to the large two-photon absorption cross-sections and the efficient electron-transfer mediated initiation of the polymerization by the $D-\pi$ - $D$ chromophores. A three-dimensional test-structure was used to evaluate how varying the intensity and the exposure times affects the shape and dimensions of the polymerization volume element (voxel). Conditions were found for which the voxel could be as small as $\sim 200 \mathrm{~nm}$ in width and $\sim 700 \mathrm{~nm}$ in length, even though the wavelength of the two-photon excitation radiation was $775 \mathrm{~nm}$.

Keywords: Two-photon induced polymerization, three-dimensional lithographic microfabrication, micro-electromechanical systems, micro-optics, microfluidics, twophoton absorption
\end{abstract}

\section{Introduction}

The process of two-photon excitation has attracted much recent attention, in part because it can afford the possibility of photo-activating a wide range of physical and chemical processes with high three-dimensional spatial resolution and tight volume confinement in a material. This feature has been the basis for several recent developments in the areas of three-dimensional imaging, optical data storage and threedimensional lithographic microfabrication (3DLM).[1-7] Using a tight-focusing excitation geometry, two-photon activated processes become strongly confined in three-dimensions because the two-photon absorption probability, that depends on the square of the input intensity, rapidly decreases with distance from the focal plane.

In 3DLM, patterned 3D-exposure by twophoton excitation can be used to locally induce polymerization or cross-linking in a resin.[6,7] The unexposed portions of the film can be removed by dissolution in a solvent (development), leaving behind the cross-linked microstructure. Relative to other microfabrication methods,[8] two-photon induced polymerization (TPIP) has the advantage that complex 3D structures can be generated in a single exposure/development cycle.

In most work to date, TPIP and 3DLM have 
been performed using photoinitiators that were developed originally for UV-excitation. As these materials do not necessarily possess large twophoton cross sections, high exposure pulse energies (or powers), sometimes rather close to the damage threshold of the material, and long exposure times are typically required. This aspect limits the versatility of TPIP and its scope for wide application. In order to increase the efficiency of TPIP, it would be useful to identify new photoinitiators that are simultaneously strong twophoton absorbers and efficient initiators.

We recently reported a design strategy for organic molecules featuring large two-photon absorption cross sections $(\delta) \cdot[9,10]$ One structural motif for these molecules is $D-\pi-D$, where $\pi$ represents a $\pi$-conjugated backbone (for example: diphenylpolyene, bis(styryl)benzene, biphenyl), and $\mathrm{D}$ is an electron donating group (for example: diphenylamino- or dibutylamino-) symmetrically substituted on the ends of the $\pi$-system. Examples of these molecules are given in Fig. 1. The maximum two-photon absorption cross section of compound 1 was found to be $200 \times 10^{-50} \mathrm{~cm}^{4}$ $\mathrm{s} /$ photon-molecule at $600 \mathrm{~nm}$. The value of $\delta$ increases with conjugation length (1300 and 1420 $\times 10^{-50} \mathrm{~cm}^{4} \mathrm{~s} /$ photon-molecule for compounds 7 and 11, respectively). Even larger values of $\delta$ have been measured for molecules with the generic structure $D-\pi-A-\pi-D$, in which electron withdrawing groups (A) are substituted on the central portion of the $\pi$-bridge.[9] These results have been interpreted in terms of a symmetric intramolecular electronic charge redistribution upon excitation that leads to large transition dipole moments between the electronic levels involved in the two-photon transition. $[9,10]$

We have shown that some of these D- $\pi$-D twophoton absorbers can be used as photoinitiators for the polymerization of acrylate monomers.[11,12] For example, chromophores 1, 8, 9, and 11 were found to initiate polymerization when incorporated in a polymerizable acrylate-based resin and irradiated with pulsed laser light at a wavelength in the two-photon absorption band. Photosensitized radical polymerization of acrylates and other monomers has been widely investigated, and a variety of processes can lead to the final production of radicals, including energy transfer, redox reactions, and interactions between the excited dye and other components of the polymerizable system.[13-17]
In the case of the $D-\pi$-D chromophores, electron transfer is believed to be involved in the process of TPIP for several reasons: (1) the twophoton chromophores exhibit excited-state fluorescence quenching in the presence of various acrylates; (2) the degree of fluorescence quenching can be correlated with the driving force for electron transfer between the D- $\pi$-D dyes and the acrylate acceptors; and (3) the radical cation of the $D-\pi$-D chromophore, in the case of $\mathbf{1}$ and $\mathbf{2}$, is generated during the photo-polymerization of acrylates. We have observed that polymerization occurs in the presence of methanol but not when excess radical scavengers are present, from which we conclude that the polymerization proceeds via a radical rather than an anionic mechanism. The polymerization threshold pulse energy (or power for mode-locked CW excitation) for TPIP using these chromophores was found to be substantially lower than that for conventional radical photoinitiators.[12,18] This suggests that properly chosen two-photon chromophores can be efficient initiators for radical polymerization. Elsewhere we have reported examples of complex 3D microstructures that were fabricated by TPIP using these initiators. $[12,18]$

In this contribution, we (1) extend our investigation to a larger class of two-photon chromophores, (2) demonstrate the versatility of the TPIP technique by presenting various complex microstructures which were prepared using these high-efficiency $D-\pi$-D initiators, and (3) describe in detail how the size of polymeric features obtained by TPIP depends on the exposure conditions, such as laser power and scan speed.

\section{Two-Photon Initiators for Polymerization}

The $D-\pi$-D two-photon absorbers that have been studied as potential initiators of radical polymerization of acrylates are listed in Fig. 1. The acrylate monomers used in this study were all kindly provided by Sartomer Co. (Exton, Pennsylvania).

Compound 1 was first found to initiate the polymerization of acrylates under two-photon excitation, and it has been extensively studied under a variety of experimental conditions. In particular, it has been shown that the intensity dependence of the polymerization rate $\left(R_{p}\right)$ in liquid acrylates under two-photon excitation is different from that under one-photon excitation (see Fig. 2; the experimental details are described later in this section).[18] $R_{p}$ depends on the 
1:

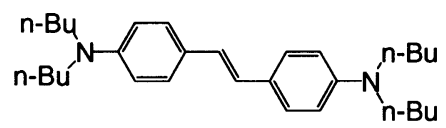

\section{2: $\quad \mathrm{R}=\mathrm{H}$ \\ 3: $\mathrm{R}=\mathrm{n}-\mathrm{Bu}$}

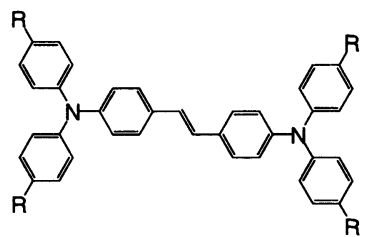

4-7: $n=2-5$

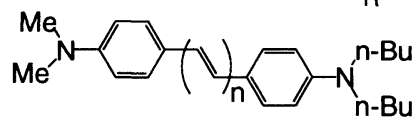

8: $\quad \mathrm{R}=\mathrm{H}$

9: $\mathrm{R}=\mathrm{OMe}$<smiles>[R]C1CC(CCc2ccc(N(CCCC)CCCC)cc2)CC(CC(C)(C)C)C1</smiles>

10: $\mathrm{R}=\mathrm{C}_{12} \mathrm{H}_{25}$

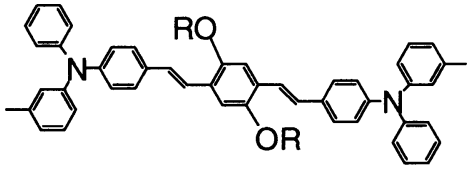

11:

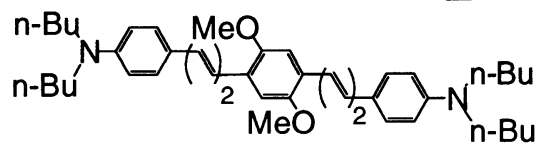

12: $\mathrm{R}_{1}=\mathrm{H}, \mathrm{R}_{2}=\mathrm{CH}_{3}$

13: $\mathrm{R}_{1}=\mathrm{OMe}, \mathrm{R}_{2}=\mathrm{CH}_{3}$

14: $R_{1}=R_{2}=H$

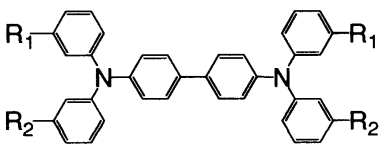

Fig. 1. Molecular structure of the chromophore investigated in this paper.

square-root of the input intensity $(I)$ in the case of one-photon excitation, but it varies linearly with intensity in the two-photon case. These findings are consistent with a polymerization process in which the initiation rate is proportional to $I$ and $I^{2}$ for one- and two-photon excitation, respectively, wherein the dominant termination process is assumed to be the bimolecular termination between propagating radicals. [13,18]

The ability of D- $\pi$-D two-photon chromophores to undergo photoinduced electron transfer toward various electron acceptors has been discussed previously.[11,12] Here we report results for all of the chromophores in Fig. 1 and for two commercial acrylate systems: SR9008 and SR454. SR9008 is an alkoxylated trifunctional acrylate ester, while SR454 is an ethoxylated trimethylolpropane triacrylate, and they are both liquids. In general, the molecules studied showed a quenching of the fluorescence emission and a shortening of the excited state lifetime in the presence of acrylate. Only in a few cases was the fluorescence quenching not accompanied by lifetime shortening (for example compound 6). The bimolecular quenching rate constant, $k_{q}$, has been determined from the change in the fluorescence intensity or lifetime as a function of the concentration of monomer, using the SternVolmer equation. $[19,20]$

The results are displayed in Fig. 3 in the form of a Marcus plot, where the values of $k_{q}$ (logarithmic scale) are plotted as a function of the free energy, $\Delta G$, of the electron transfer reaction. $\Delta G$ can be written in the following way, neglecting the Columbic work term: [21]

$$
\Delta G=E\left(D_{o n}^{+} / D o n\right)-E\left(A c c / A c c^{-}\right)-E_{e x c},
$$

where $E\left(\right.$ Don $\left.^{+} / D o n\right)$ is the electrode potential of the electron donor (the two-photon chromophore in this case), $E\left(A c c / A c c^{-}\right)$is the electrode potential of the electron acceptor (the acrylate), and $E_{e x c}$ is the energy difference between the ground state $\left(S_{0}\right)$ and the lowest-lying excited state $\left(\mathrm{S}_{1}\right)$ of the twophoton chromophore. Fig. 3 includes the results for the SR9008 acrylate system (obtained from both steady-state fluorescence and lifetime measurements), and for SR454 (from steady-state fluorescence only). The reduction potentials for the acrylates are: $-2.770 \mathrm{~V}$ for SR9008 and -2.695 $\mathrm{V}$ for SR454 (for a comparison, the corresponding value for methyl methacrylate is $-2.980 \mathrm{~V}$. All values are referenced to ferrocenium/ferrocene in THF / $0.1 \mathrm{M}\left[\mathrm{Bu}_{4} \mathrm{~N}\right]^{+}\left[\mathrm{PF}_{6}\right]^{-}$. The lifetime of the chromophores (in acetonitrile) is in the range 0.2-2 $\mathrm{ns}$ in the absence of the quencher. As can be seen from Fig. 3, the experimental data are roughly distributed along a parabolic curve, as predicted by Marcus theory.[21,22] The peak of the parabola occurs roughly at $\Delta G \approx-0.8 \mathrm{eV}$, which is

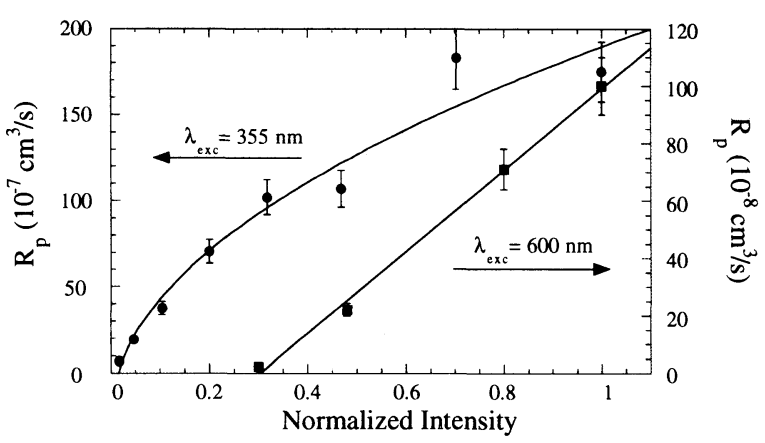

Fig. 2. Polymerization rate of the acrylate SR9008 with compound 1 as initiator for one-photon excitation at 355 $\mathrm{nm}(\boldsymbol{)})$ and two-photon excitation at $600 \mathrm{~nm}(\boldsymbol{\square})$. The intensity scale has been normalized to the maximum intensity used in each case. 
consistent with typical values of the reorganization energy in polar solvents.[23] Most of the twophoton chromophores included in this study show a negative $\Delta G$ value -- that is the photoinduced electron transfer is thermodynamically favorable. But also chromophores for which $\Delta G>0$ show substantial quenching constants $\left(k_{q}>1 \times 10^{8}\right.$ $\mathbf{M}^{-1} \mathbf{s}^{-1}$.

It should be noted that the data points for chromophores $1,4,12$, and 13 lie relatively close to the maximum of the parabola, suggesting that they are good candidates as photoinitiators of acrylate polymerization. However, all the chromophores in Fig. 1 (except 3 and 10, which have not yet been tested, but should behave similarly to the other chromophores in the series) have been found to initiate polymerization of acrylates, even if $\Delta G>0$. Of particular note, chromophores 12 and 13 exhibit a strong twophoton absorption peak near $525 \mathrm{~nm}$, that is blueshifted with respect to that of the $D-\pi-D$ chromophores 1-11, due to the short length of the conjugated $\pi$-bridge.[24] This characteristic can be exploited in microfabrication to obtain smaller features, because the size of the voxel depends on the wavelength of the laser light.

The rate of polymerization, $R_{p}$, of the acrylate SR9008 (after removal of the inhibitor MEHQ)

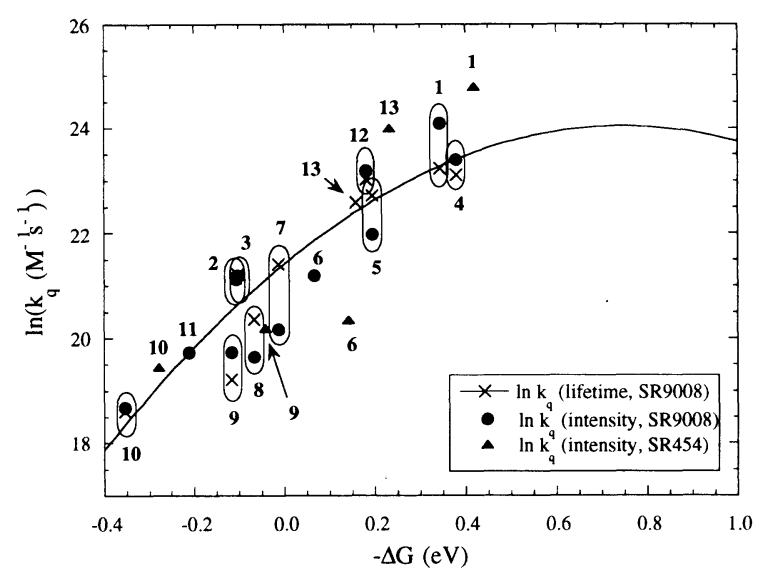

Fig. 3. Marcus plot for the bimolecular quenching rate constants $k_{q}$ as a function of $-\Delta G$. $\times$ : quencher $=$ SR9008, method = fluorescence lifetime (the line represents a parabolic fit to this set of data); quencher $=$ SR9008, method $=$ steady-state fluorescence intensity; $\mathbf{\Delta}$ : quencher = SR454, method = steady-state fluorescence intensity. In all cases, the solvent was acetonitrile. The data points are labeled with the chromophore number (see Fig. 1) and the ovals enclose the two data points relative to the SR9008 acrylate for each chromophore (where available). and its dependence on the input laser power was measured for some of the two-photon dyes in Fig. 1 in a dose array experiment. A solution of the dye in the neat monomer was exposed to pulsed laser light for various time intervals and input powers. After washing away the unpolymerized monomer, the polymerized features were imaged using scanning electron microscopy and their volumes were estimated. Fig. 4 shows the results for compounds 12 and 13. In this case the wavelength of the excitation source was $532 \mathrm{~nm}(8-\mathrm{ns}, 10-\mathrm{Hz}$ pulses), and the beam was collimated through the sample with a diameter of approximately $225 \mu \mathrm{m}$. The concentration of two-photon dye in the neat SR9008 monomer was about $5 \mathrm{mM}$. Each data point in the figure represents the polymerization rate at a certain input power, determined from the slope of a plot of the volume of the polymerized features as a function of exposure time (1-30 s). Similar to the case of $\mathbf{1}$ discussed above, the rates reported in Fig. 4 vary approximately linearly with laser power, in agreement with a polymerization mechanism that involves two-photon absorption in the initiation step.[18]

The availability of a wide selection of twophoton absorbers that can be used as photoinitiators offers several advantages. First of all, the maximum of the two-photon absorption spectrum for the chromophores in Fig. 1 occurs over a broad wavelength range, from about $530 \mathrm{~nm}$ to $775 \mathrm{~nm} \cdot[9,10]$ This means that these initiators allow a wide variety of laser sources to be used for high-efficiency TPIP, as required for a specific application. For example, CW mode-locked Ti:sapphire lasers are a convenient, commercially

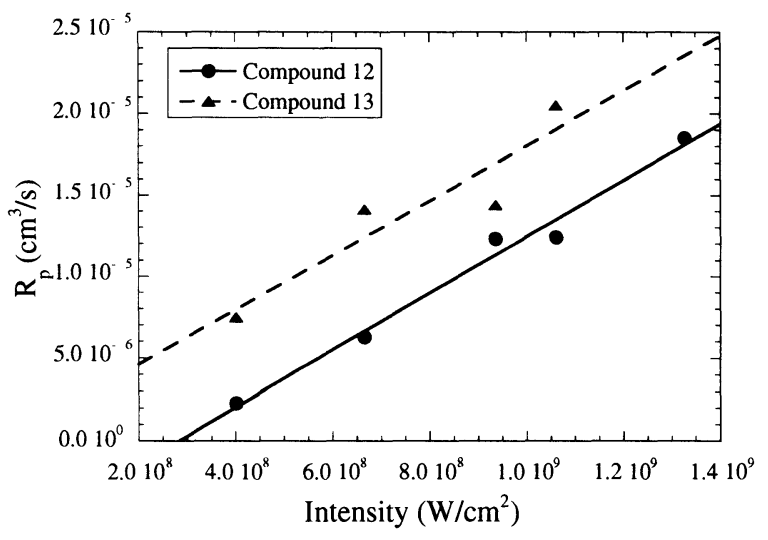

Fig. 4. Polymerization rate $R_{p}$ of SR9008 in the presence of compounds 12 and $\mathbf{1 3}$ as photoinitiators after exposure to $532 \mathrm{~nm}$ laser light of various intensities. 
available source of high-intensity, high-repetition rate laser pulses from $690-1050 \mathrm{~nm}$, and as such are well suited for microfabrication by TPIP. Use of this laser system for 3DLM is made more practical by the availability of efficient two-photon initiators, such as 11, which exhibits peak twophoton absorption at $775 \mathrm{~nm}$. A chromophore could also be chosen for use with a specific acrylate based on the position of its excited state with respect to the electronic levels of the monomer, to optimize (or, more generally, control) the electron transfer rate.

\section{Microfabrication by TPIP}

The technology of 3DLM [4-6,12,25-28] is promising in that complex 3D microstructures, which would be either difficult or time-consuming to fabricate by any other technique, can be produced in a single step. Previously, this approach was of limited use since the available initiators were weak two-photon absorbers, and therefore high-power excitation sources were required for TPIP. The enhanced two-photon sensitivity of the D- $\pi$-D initiators described here offers a means for preparing high-sensitivity TPIP resins, which should allow 3DLM to be applied more widely. Efficient 3DLM resins could also be

(a)
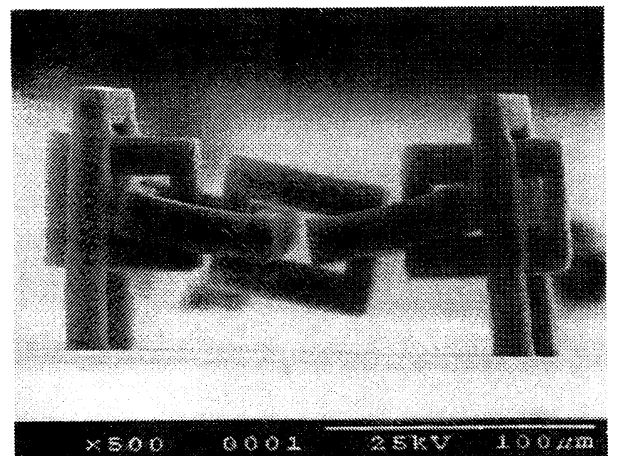

(b)

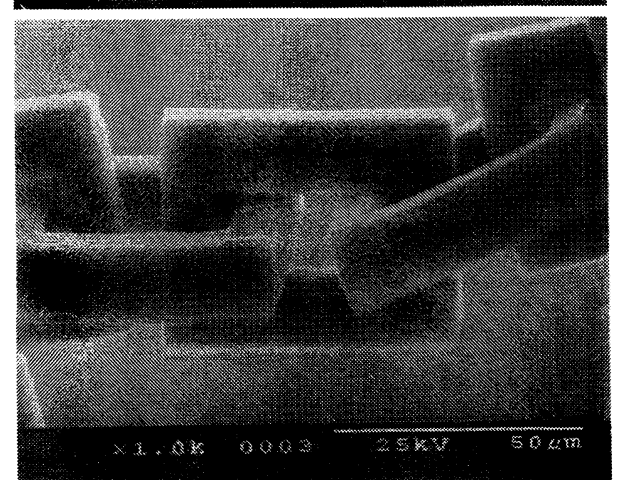

Fig. 5. (a) SEM image of a micro-chain links obtained by TPIP (laser power: $6.6 \mathrm{~mW}$, excitation wavelength: $800 \mathrm{~nm}$; two-photon initiator: 11). (b) Close-up of the central links in (a). useful for certain types of 3D optical data storage. $[2,3,12]$

To demonstrate the utility of these two-photon initiators, we formulated two-photon cross-linkable semi-solid acrylate resins and manufactured a range of structures by 3DLM. The two-photon resins consisted, by weight, of $0.1 \%$ initiator 9 or $11,70 \%$ multifunctional acrylate monomer, and $29.9 \%$ poly(styrene-co-acrylonitrile) as a polymer binder. The monomer components were typically 50\% SR368 (inhibitor present) and 50\% SR9008 (inhibitor removed) and were chosen for their surface adhesion and mechanical strength properties. The resin mixture was dissolved in dioxane, cast onto glass substrates at a controlled thickness, and allowed to set by slow evaporation of the solvent. The thickness of the cured films could be varied from $100-200 \mu \mathrm{m}$. Cured films were exposed by irradiation with tightly focused laser pulses (N.A. $=1.4, \sim 0.35-\mu \mathrm{m}$ radial spot size) from a Ti:sapphire laser, that was tuned into the two-photon absorption band of the D- $\pi$-D initiator (730 $\mathrm{nm}$ for $9 ; 800 \mathrm{~nm}$ for 11$)$. Over the course of our investigations, two different commercial lasers have been used. One of these produces 150-fs pulses at a $76 \mathrm{MHz}$ repetition rate (Coherent Mira),

(a)

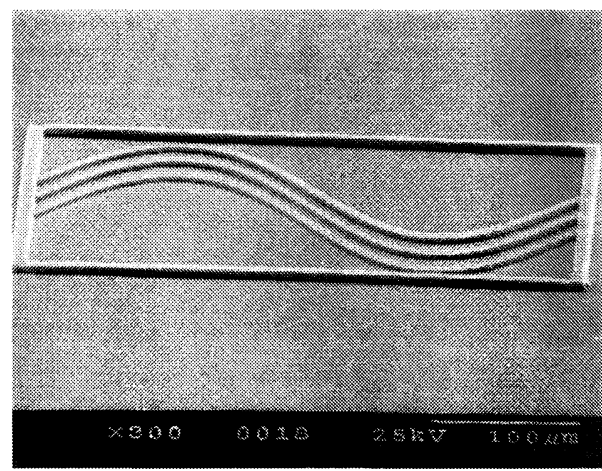

(b)

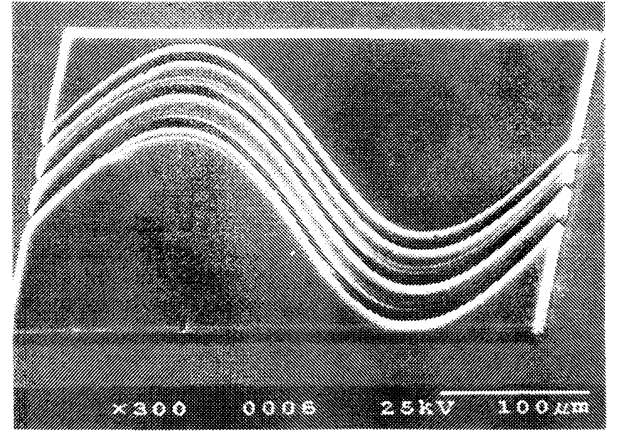

Fig. 6. SEM images microstructures obtained by TPIP. (a) Three sinusoidal ridges (laser power: $5.6 \mathrm{~mW}$, excitation wavelength: $800 \mathrm{~nm}$; two-photon initiator: 11). The ridges are about $20 \mu \mathrm{m}$ apart. (b) Sinusoidal channels (laser power: $5 \mathrm{~mW}$, excitation wavelength: $775 \mathrm{~nm}$; two-photon initiator: 11). 
whereas the other generates 85 -fs pulses at a repetition rate of $82 \mathrm{MHz}$ (Spectra-Physics Tsunami). The exposures were patterned under computer-control by shuttering the beam and translating the film relative to the focal point using a 3-axis micropositioner (Sutter MP-285). Following exposure, the film was immersed in dimethylformamide to dissolve the non-irradiated resin leaving behind the cross-linked 3D structure.

Figs. 5 and 6 show examples of the microstructures fabricated by the above method. Fig. 5 is a scanning-electron micrograph (SEM) of a set of free-standing inter-locked microchain links. The chains were fabricated using a power of $6.6 \mathrm{~mW}$ and a translation speed of $50 \mu \mathrm{m} / \mathrm{s}$. Taking the voxel width to be the diameter of the focused beam $(\sim 0.70 \mu \mathrm{m})$, the effective exposure time per voxel was approximately $14 \mathrm{~ms}$. The dimensions of the individual links as designed (as observed) are: length $=60 \mu \mathrm{m}(59 \mu \mathrm{m})$, height $=$ $44 \mu \mathrm{m}(46 \mu \mathrm{m})$, and thickness $=8 \mu \mathrm{m}(10-16 \mu \mathrm{m})$. Inspection of Fig. 5 reveals that the chain links are freely movable; note in particular that the central link has relaxed to a sloped position after development. The microchain illustrates the versatility of TPIP and its potential as a fabrication tool for manufacturing microstructures containing inter-locked parts that can be moved or otherwise acted upon independently, as is essential for microelectromechanical systems (MEMS) and microfluidics. $[8,29]$

It is well known that volume shrinkage occurs during the polymerization of acrylates and during the development phase, which can appreciably alter the dimensions and form of the structure.[30,31] Accounting for these factors, and in particular, designing material systems which minimize the effects of shrinkage and distortion will greatly facilitate the development of TPIP into an widely-applied microfabrication technique. Currently, we are studying a variety of composite material systems designed to optimize TPIP of acrylates. The size of the voxel as a function of irradiation-power and write-speed (see below) was not accounted for in the design of the microchain, and to a lesser extent, this may contribute to some of the differences between the designed and observed dimensions.

Fig. 6a shows a microstructure composed of a set of raised sinusoidal ridges. The complement of this structure was also fabricated and is shown in Fig. 6b. These images demonstrate how versatile TPIP can be for producing micro-parts, templates, and structures of arbitrary form. The chain-links of Fig. 5 are highly complex in terms of the amount of "under-cut", and inter-connectivity of the components. Producing this structure by conventional two-dimensional mask exposures would be difficult, because it would necessarily require many steps, whereas the structure can be fabricated by TPIP using a single exposure. The ability to fabricate structures containing microchannels (like the ones shown in Fig. 6) could be exploited in microfluidics applications.

\section{Sensitivity}

Earlier and in the previous section, we demonstrated how these two-photon initiators and nonlinear photopolymer resins could be used to fabricate a wide range of structures which are of interest in a number of emerging technologies.[12] These include micro-optical components, such as tapered wave-guides, diffraction gratings, and photonic band-gaps, and micro-mechanical devices, such as cantilever structures. An important parameter that needs to be optimized in the development of this technology is the sensitivity of the photo-polymer system. Lower powers and shorter exposure times can be used to fabricate a desired structure as the resin is made more sensitive.

We compared the TPIP sensitivity of a range of semi-solid photopolymer resins exposed with femtosecond pulses at $730-800 \mathrm{~nm}$. Films of the resins were prepared as described in Section 3 and included the D- $\pi$-D chromophore 9 or 11 or the UV radical generators $\mathbf{1 5 - 1 7}$ as the photoinitiator (see Tab. 1). The resins were exposed at wavelengths near the two-photon absorption peak of the D- $\pi$-D initiators. The threshold power for writing $\left(P_{t h}\right)$ was defined as that power for which a polymeric structure could be fabricated that was adequately cross-linked so as to survive the developing stage. $P_{t h}$ was found to be $200 \mu \mathrm{W}$ for 9 at $730 \mathrm{~nm}$ and $300 \mu \mathrm{W}$ for 11 at $800 \mathrm{~nm}$. In both cases, damage was observed at powers greater than $10 \mathrm{~mW}\left(P_{D}\right)$. The dynamic power range for polymerization $\left(P_{D} / P_{t h}\right)$ is then $\sim 50$ and 33 for 9 and 11 , respectively (Table 1 ). In the absence of initiator, no polymerization occurred at powers below the damage threshold. The films with 15-17 as photoinitiators were found to have much smaller dynamic ranges, varying from $\sim 1$ to 2.5 at $730 \mathrm{~nm}$. The fact that $P_{t h}$ is large and close to $P_{D}$ for these UV-initiators means that damage can easily occur during the fabrication process. Moreover, the 
Table 1. Sensitivity data for photo-polymerizable resins containing various initiators. $P_{t h}$ is the power threshold for writing (see text); $P_{D}$ is the power at which the resin film is damaged. The ratio $P_{D} / P_{t h}$ represents the dynamic range for the resin. The resins were exposed to highly focused laser pulses $(\approx 0.35 \mu \mathrm{m}$ radial spot size, $150 \mathrm{fs}, 76 \mathrm{MHz})$ at 730 $\mathrm{nm}(800 \mathrm{~nm}$ in the case of $\mathbf{1 1})$.

\begin{tabular}{cccc}
\hline & $P_{t h} / \mathrm{mW}$ & $P_{D} / \mathrm{mW}$ & Dynamic range \\
\hline
\end{tabular}

degree of cross-linking that can be achieved for powers just above $P_{t h}$ could be too low to prevent deformation of the structure during development. As a consequence, it is difficult to fabricate microstructures in a reliable way using such UVinitiators, and there is limited flexibility in terms of controlling the degree of cross-linking and thus the mechanical properties of the target structure. A large $P_{t h}$ also affects the complexity of the structures that can be obtained, because the voxel dimensions depend on the excitation power (see below). Replacing the UV-initiator (15-17) with a $D-\pi$-D chromophore, which is designed to have large two-photon absorptivity, has resulted in photopolymers with more than an order-ofmagnitude improvement in TPIP photosensitivity and thus has greatly increased the potential of the TPIP technique.

\section{Optimizing two-photon microfabrication}

In general, controlling the microstructure properties, such as mechanical strength, dimension of the smallest features, complexity, and repeatability, can be achieved by proper choice of writing speed, voxel size, power, sensitivity of the resin, and other parameters of the TPIP process. The features that we have photopolymerized by two-photon absorption are comparable in size to those reported earlier. However, because of the improved sensitivity of the two-photon initiators used here as compared to conventional materials, the speed at which these features can be produced is increased. This is particularly important in optical data storage, where large numbers of bits need to be recorded quickly or in the utilization of 3DLM as a fabrication tool for mass production. This potential of the TPIP method is illustrated in Fig. 7, that shows a structure of dimensions 180 $\mu \mathrm{m} \times 180 \mu \mathrm{m} \times 20 \mu \mathrm{m}$, obtained as follows. The sample was held fixed relative to the focusing lens and the laser beam was passed through a confocal scan-head (Bio-Rad MRC 1024) in which the beam can be rastered in two dimensions in the focal plane of the objective, by deflection from galvo-driven mirrors. The scan speed used to fabricate this structure was $9 \mathrm{~cm} / \mathrm{s}$ (the scanning direction is easily identified by inspection of Fig. $7 \mathrm{~b}$ and the spacing between adjacent lines is $0.35 \mu \mathrm{m})$. This speed is much larger than those used for the fabrication of the other structures presented in this paper, obtained by moving the film (with speeds of the order of $50 \mu \mathrm{m} / \mathrm{s}$ ) and keeping the laser beam fixed. The $3 \mathrm{D}$ object is obtained by sequentially moving the focal plane into the sample by $0.2 \mu \mathrm{m}$ after each layer is rastered. The total writing time for this structure was of $100 \mathrm{~s}$. The close-up of the structure in Fig. $7 \mathrm{~b}$ demonstrates the smoothness and reproducibility of the polymerized features, that are predicated on the high sensitivity of the starting polymerizable resin. The left portion of the structure appears raised from the substrate, 
probably because of poor adhesion of the polymer to the glass substrate or a slight tilt between the substrate itself and the plane in which the beam was moved.

Shrinkage of the polymerized resin during development can affect the size and appearance of the microstructures obtained with this and other techniques. In some cases, the problem can be solved by using different polymerizable acrylates (or other types of monomers) that show a smaller degree of shrinkage.[30,31] On the other hand, the mechanical properties of the acrylates investigated here (or similar ones) could be desirable for certain applications. In order to control the shape and size of the final product it is then necessary to characterize in detail how the parameters of the writing and subsequent development processes affect the size of the voxels that build up the final product.

A test structure was designed and fabricated to study the shape and dimensions of the polymerization voxel as a function of exposure conditions. The test structure, shown in Fig. 8, was fabricated using the same composition for the two-photon resin as described in Section 3, with compound 11 as photoinitiator and an excitation

(a)

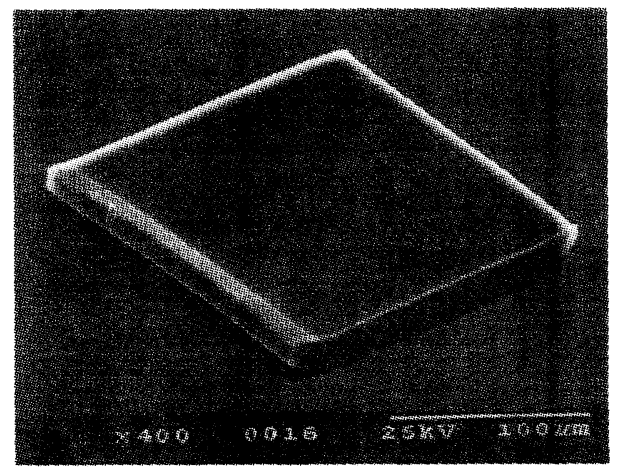

(b)

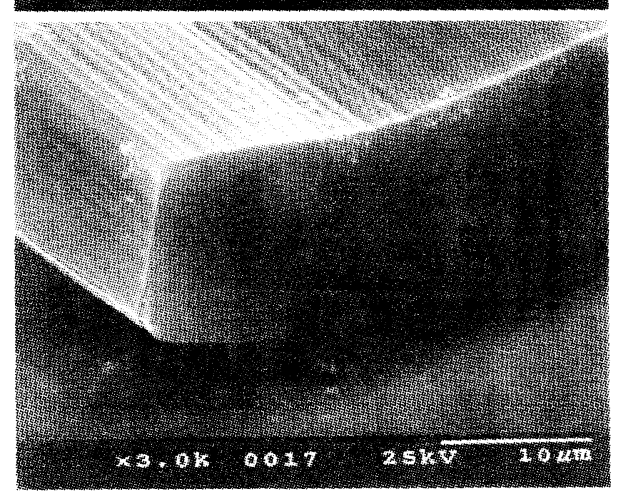

Fig. 7. (a) SEM image of a structure obtained using TPIP by rastering a laser beam of $3 \mathrm{~mW}$ at scan speed of $9 \mathrm{~cm} / \mathrm{s}$ (excitation wavelength: $775 \mathrm{~nm}$; two-photon initiator: 11). The lateral dimension of the square is about $180 \mu \mathrm{m}$. (b) Close-up of the structure in (a). wavelength of $775 \mathrm{~nm}$. The microstructure consists of a block of polymer designed to be 170 $\mu \mathrm{m}$ in length, $20 \mu \mathrm{m}$ in width and about $24 \mu \mathrm{m}$ in height, built by scanning the resin with the laser beam along lines parallel to the substrate and in the width direction. The laser power used for the fabrication of this portion was $2.75 \mathrm{~mW}$ and the writing speed was $100 \mu \mathrm{m} / \mathrm{s}$. The actual dimensions of the final product are: $164 \mu \mathrm{m}$ (length), $22 \mu \mathrm{m}$ (width), $28 \mu \mathrm{m}$ (height). At a fixed height, a set of lines was written in such a way that the lines extend by $3 \mu \mathrm{m}$ on both sides of the structure. As can be seen from Fig. 8a (top view) and $8 \mathrm{~b}$ (side view), this results in linear features that protrude from the main body of the microstructure. These lines are spaced by about 10 $\mu \mathrm{m}$ and each one is written at a different scan speed (ranging from 1 to $180 \mu \mathrm{m} / \mathrm{s}$, with speeds increasing from the right to the left side of the structure in the Fig. 8a). At low scan speeds (1-5 $\mu \mathrm{m} / \mathrm{s})$, discontinuous motion of the micropositioner caused the lines to be nonuniformly exposed such that some of the lines

(a)

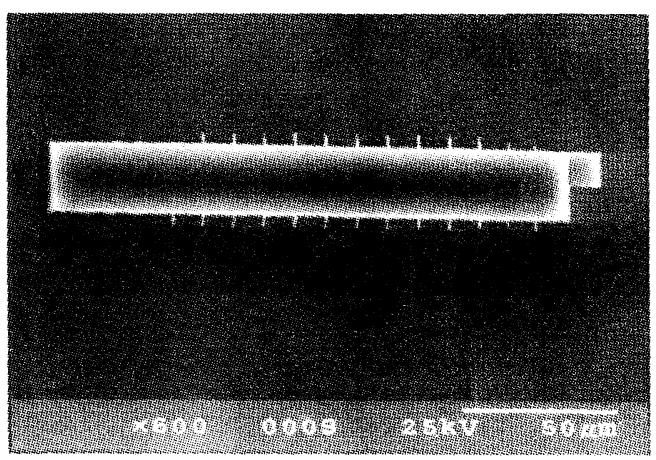

(b)

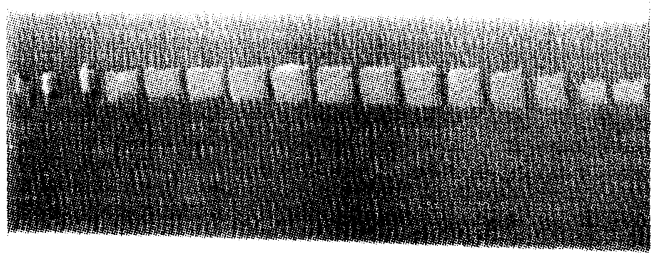

Fig. 8. (a) SEM image (top view) of the microstructure fabricated by TPIP and utilized to study the dependence of the voxel size on writing speed and laser power. The lines are written with increasing scan speed from the right to the left of the image (in the range from 1 to 180 $\mu \mathrm{m} / \mathrm{s}$ ). The power used for writing this set of lines was $0.825 \mathrm{~mW}$, while for the parallelepiped it was $2.75 \mathrm{~mW}$. (b) Side view of the structure and lines in (a). 


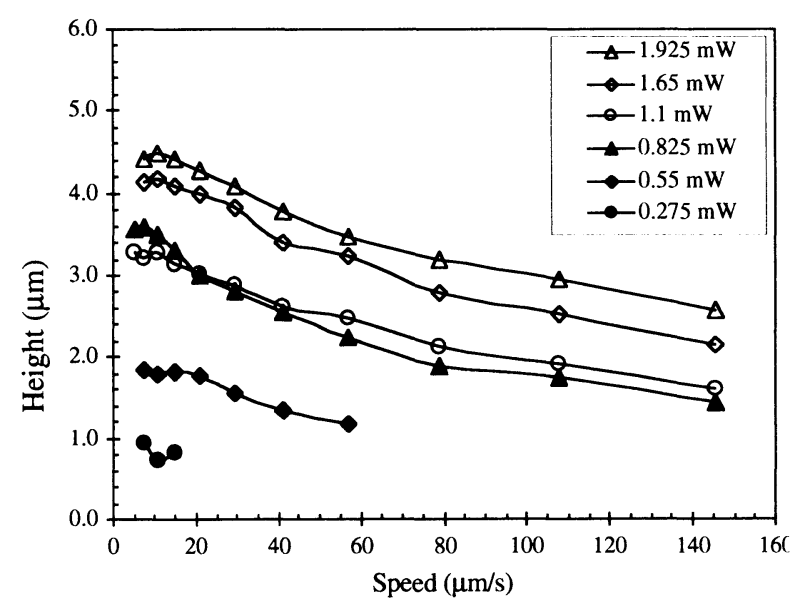

Fig. 9. Height of the written protruding lines for test structures such as the one in Fig. 8, as a function of scan speed and input laser power.

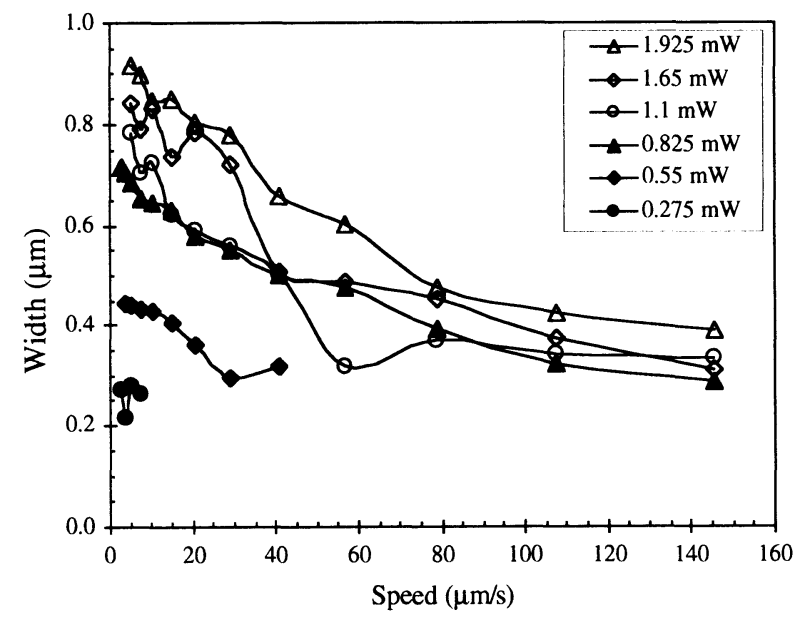

Fig. 10. Width of the written protruding lines for the test structures such as the one in Fig. 8, as a function of scan speed and input laser power.

are deformed or do not survive the development process. These lines are not included in the analysis presented below. An array of these test structures (parallelepiped and lines) was then fabricated, in which only one parameter was varied, namely the power at which the protruding lines were written (from 0.275 to $1.925 \mathrm{~mW}$; the power at which the parallelepiped was generated was instead always kept fixed). The little square feature (about $10 \mu \mathrm{m}$ in size) visible in the right side of the parallelepiped in Fig. 8a was generated to introduce an asymmetry in the structure for easy identification of the lines.

It is immediately evident that the dimensions of the protruding lines are different for the various speeds. Increasing the scan speed results in a reduction in the effective exposure (or dwell) time for each voxel, and consequently in a lower number of radicals generated locally and in a smaller degree of cross-linking in the resulting polymer. As a consequence, the final features become smaller with increasing scan speed and a limiting speed for writing is generally observed. If the power of the excitation beam is reduced, we can similarly expect that the voxel size would be smaller, because the region where the local intensity of the beam exceeds the threshold value is smaller. The dimensions of the linear features were determined through the analysis of SEM images of the structures. The results are reported in Fig. 9 for the heights and in Fig. 10 for the widths. In both cases the feature dimensions gradually decrease with increasing scan speed. For a fixed scan speed, both the height and the width of the lines increases with increasing input power, as expected. The smallest features that were obtained during this set of experiment are about $200 \mathrm{~nm}$ wide and $700 \mathrm{~nm}$ high. The results in Figs. 9 and 10 indicate that, for a given laser power and scan speed, the height of a written line is much larger than its width (by approximately a factor of 4), meaning that a better confinement of the excitation can be obtained in the plane perpendicular to the direction of propagation of the beam than along the propagation axis. This also explains the spread in thicknesses reported for the chain links of Fig. 5 (see Section 3): the thickness was defined to be 8 $\mu \mathrm{m}$ in all cases, but the observed values vary from 10 to $16 \mu \mathrm{m}$, depending on the direction in which the resin was moved with respect to the beam to write each portion of the links.

If we assume that the voxels have cylindrical symmetry, as expected in the case of a focussed Gaussian beam or any beam with a radially symmetric spatial profile, the volume of each voxel can be approximated by the volume of the ellipsoid of rotation with one axis equal to the height, and the two equivalent axes equal to the width of the linear features described above. The volumes obtained in this way are displayed in Fig. 11 for the various laser powers as a function of the inverse of the scan speed (which is proportional to the exposure time of the single voxel). Consistent with the trend shown by the data presented in Figs. 9 and 10, the voxel volume increases when the input power or the exposure time increases and seems to saturate at large times. The data can be reasonably fitted by an equation of the form:

$$
V=A[1-\exp (-B \cdot X)]
$$




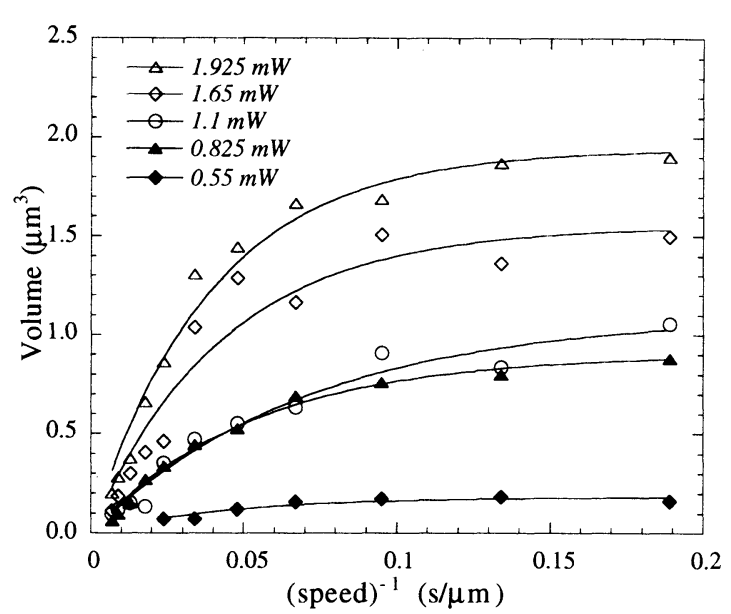

Fig. 11. Volume of the polymerized voxels as a function of the inverse of the scan speed for various laser powers. The shape of the voxel is assumed to be an ellipsoid of rotation (see text).

where $V$ is the volume, $X$ the inverse of the scan speed, and $A$ and $B$ are two fitting parameters. The rate of polymer growth can be estimated from the rise of the exponential for short exposure times, which is proportional to $G_{p}=A \cdot B$. An analysis of $G_{p}$ as a function of power can yield the polymerization threshold power, $P_{t h}$, and the power-dependence, $n$, of the photopolymerization: $G_{p} \propto\left(P-P_{t h}\right)^{n}$. The intensity-dependence can also provide valuable insight into the mechanism and quantum efficiency of the photoinitiation, as well as the mechanism of the polymerization reaction. Consistent with expectation, we find that $G_{p}$ increases with increasing intensity, although these particular data were not of sufficient quality to extract a definitive value for $n$. We are currently undertaking additional experiments to further our understanding in this area.

In addition to affecting the size and shape of the voxel, the exposure conditions will also determine the degree of cross-linking within the polymer matrix, and therefore the mechanical properties of the structure. In certain applications, particularly those for which the microstructure has mechanical functionality, the choice of exposure conditions may be dictated by not only the feature resolution and surface roughness that is sought, but also the mechanical properties which are needed for proper function, such as strength, elasticity, and yield.

The above study illustrates that sub-excitationwavelength sized features can be obtained through careful control of the exposure conditions. As an example, Fig. 12 shows a photonic crystal-type "stack of logs" structure[12] having an average (a)

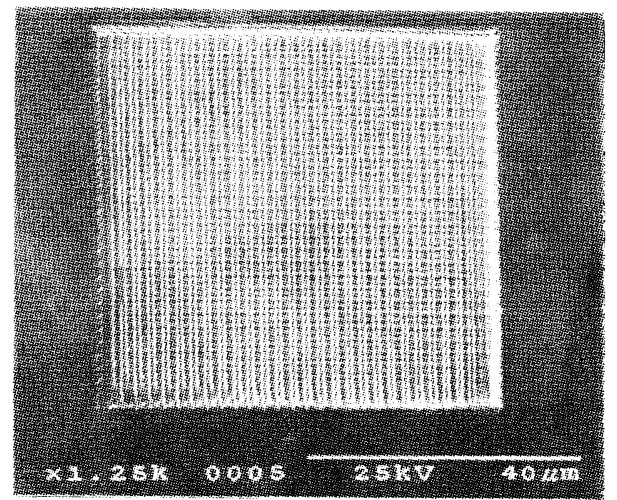

(b)

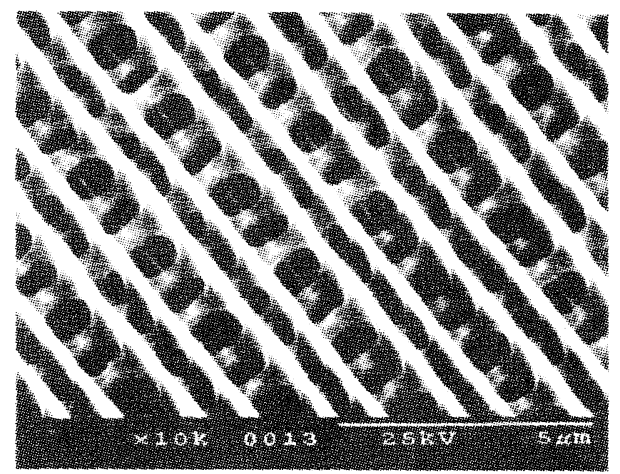

Fig. 12. (a) SEM image of a photonic band gap crystal obtained by TPIP. Excitation wavelength: $730 \mathrm{~nm}$; laser power: $0.45 \mathrm{~mW}$; scan speed: $50 \mu \mathrm{m} / \mathrm{s}$; two-photon initiator: 10. (b) Close-up of the structure in (a). The average periodicity is $1 \mu \mathrm{m}$ and the line width $0.2 \mu \mathrm{m}$.

periodicity of $1 \mu \mathrm{m}$, a base area of $60 \mu \mathrm{m}$ by 60 $\mu \mathrm{m}$, and a height of $8 \mu \mathrm{m}$. The structure was obtained by writing nine layers of $1-\mu \mathrm{m}$ spaced parallel polymer rods, with a layer spacing of 1 $\mu \mathrm{m}$, where the rods of adjacent layers were written in perpendicular directions. As can be seen from the close-up (Fig. 12b), the lines that comprise the structure are about 200-nm wide, which is much smaller than the fabrication wavelength of 730 $\mathrm{nm}$. These feature sizes are comparable to those reported by Maruo and Ikuta.[29] It should be noted that the size of the voxel can be further reduced by using high-sensitivity two-photon initiators that can be excited at shorter wavelength, such as 12-14. Knowledge of how to control the voxel size and how to obtain reproducible features should expand the potential of TPIP as a microfabrication tool.

\section{Conclusions}

TPIP provides a versatile method for fabricating complex three-dimensional microstructures in a single processing step. Structures having over-hangs, void spaces, freely-movable parts, topologically complex interconnections and 
a wide range of size-scales can be prepared without the need for multi-step processing. However, if TPIP is to become a widely applied manufacturing process, material systems and methods must be developed which permit low-power, high-speed fabrication of high-fidelity structures from a variety of media. We have taken steps in this direction by designing and synthesizing highsensitivity radical initiators which can be activated by two-photon excitation across a wide range of wavelengths $(530-800 \mathrm{~nm})$. Resins formulated with these two-photon initiators exhibit lower polymerization thresholds and higher-sensitivity than those based on conventional UV initiators. Using these new materials, we have demonstrated the potential and versatility of TPIP for micromanufacturing by fabricating various complex three-dimensional microstructures. We have shown that the size of the polymerization voxel -and thus the smallest obtainable features -- can be varied by carefully controlling the exposure time and intensity. Under certain conditions, features with dimensions much smaller than the wavelength of the two-photon excitation radiation are achievable. We believe that the applications of TPIP can be expanded by developing new multiphoton-absorbing photoactive materials.

\section{Acknowledgements}

Support of this work by the Office of Naval Research (through CAMP), the National Science Foundation (Chemistry Division) and the Air Force Office of Scientific Research (through LC-MURI) is gratefully acknowledged. We thank D. McCordMaughon and $H$. Röckel for many useful discussions.

\section{References}

1. W. Denk, J. H. Strickler and W. W. Webb, Science 248 (1990) 73.

2. J. H. Strickler and W. W. Webb, Opt. Lett. 16 (1991) 1780.

3. D. A. Parthenopoulos and P. M. Rentzepis, Science 245 (1989) 843.

4. J. H. Strickler and W. W. Webb, Proc. SPIE (1990) Vol. 1398, 107.

5. E. S. Wu, J. H. Strickler, W. R. Harrell and W. W. Webb, Proc. SPIE (1992), Vol. 1674, 776.

6. S. Maruo, O. Nakamura and S. Kawata, Opt. Lett. 22 (1997) 132.

7. S. Maruo and S. Kawata, J. MEMS 7 (1998) 411.

8. P. Rai-Choudhury, Ed., "Handbook of
Microlithography, Micromachining, \& Microfabrication", SPIE Press and The Institute of Electrical Engineers, Bellingham, WA (1997), Vol. 2.

9. M. Albota, D. Beljonne, J.-L. Brédas, J. E. Ehrlich, J.-Y. Fu, A. A. Heikal, S. E. Hess, T. Kogej, M. D. Levin, S. R. Marder, D. McCordMaughon, J. W. Perry, H. Röckel, M. Rumi, G. Subramaniam, W. W. Webb, X.-L. Wu and C. Xu, Science 281 (1998) 1653.

10. M. Rumi, J. E. Ehrlich, A. A. Heikal, J. W. Perry, S. Barlow, Z. Hu, D. McCord-Maughon, T. C. Parker, H. Röckel, S. Thayumanavan, S. Marder, R., D. Beljonne and J.-L. Brédas, J. Am. Chem. Soc. 122 (2000) 9500.

11. B. H. Cumpston, J. E. Ehrlich, L. L. Erskine, A. A. Heikal, Z.-Y. Hu, I.-Y. S. Lee, M. D. Levin, S. R. Marder, D. J. McCord, J. W. Perry, H. Röckel, M. Rumi and X.-L. Wu, in "Mat. Res. Soc. Symp. Proc. - Electrical, Optical, and Magnetic Properties of Organic Solid-State Materials IV", Reynolds, J. R., Jen, A. K.-Y., Rubner, M. F., Chiang, L. Y. and Dalton, L. R., Eds., MRS, Warrendale (1998), Vol. 488, p. 217.

12. B. H. Cumpston, S. Ananthavel, S. Barlow, D. L. Dyer, J. E. Ehrlich, L. L. Erskine, A. A. Heikal, S. M. Kuebler, I.-Y. S. Lee, D. McCord-Maughon, J. Qin, H. Röckel, M. Rumi, X.-L. Wu, S. R. Marder and J. W. Perry, Nature 398 (1999) 51.

13. G. Odian, "Principles of Polymerization", 2nd ed., John Wiley \& Sons, New York (1981).

14. G. Oster and N.-L. Yang, in "Selected Papers on Photopolymers; Physics, Chemistry, and Applications", Lessard, R. A. and Manivannan, G., Eds., SPIE Optical Engineering Press, Bellingham (1968), Vol. MS 114, p. 10.

15. F. Takemura, Bull. Chem. Soc. Jap. 35 (1062) 1073.

16. F. Takemura, Bull. Chem. Soc. Jap. 35 (1962) 1078.

17. C. Roffey, "Photogeneration of Reactive Species for UV Curing", Wiley, New York (1997).

18. S. M. Kuebler, B. H. Cumpston, S. Ananthavel, S. Barlow, J. E. Ehlrich, L. L. Erskine, A. A. Heikal, D. McCord-Maughon, J. Qin, H. Röckel, M. Rumi, S. R. Marder and J. W. Perry, Proc. SPIE (2000), Vol. 3937, 97.

19. J. N. Demas, "Excited State Lifetime Measurements", Academic Press, New York (1983).

20. N. J. Turro, "Molecular Photochemistry", W. A. Benjamin, Inc., Reading, Massachussetts (1965). 
21. G. J. Kavarnos and N. J. Turro, Chem. Rev. 86 (1986) 401.

22. R. A. Marcus and N. Sutin, Biochim. Biophys. Acta 811 (1985) 265.

23. J. R. Bolton and M. D. Archer, in "Electron Transfer in Inorganic, Organic and Biological Systems", Bolton, J. R., Mataga, N. and McLendon, G., Eds., American Chemical Society, Washington, D. C. (1991) Vol. 228, p. 7.

24. J. E. Ehrlich, S. P. Ananthavel, S. Barlow, K. Mansour, K. Mohanalingam, S. R. Marder, J. W. Perry, M. Rumi and S. Thayumanavan, Nonlinar Optics, in press.

25. M. P. Joshi, H. E. Pudavar, J. Swiatkiewicz, P. N. Prasad and B. A. Reinhardt, Appl. Phys. Lett. 74 (1999) 170.
26. G. Witzgall, R. Vrijen, E. Yablonovitch, V. Doan and B. J. Schwartz, Opt. Lett. 23 (1998) 1745.

27. S. Maruo and K. Ikuta, Appl. Phys. Lett. 76 (2000) 2656.

28. H.-B. Sun, T. Kawakami, Y. Xu, J.-Y. Ye, S. Matuso, H. Misawa, M. Miwa and R. Kaneko, Opt. Lett. 25 (2000) 1110.

29. S. Maruo and K. Ikuta, Proc. SPIE (2000) Vol. 3937, 106.

30. H. Tagoshi and T. Endo, J. Appl. Polym. Sci. 43 (1991) 1933.

31. P. Karrer, S. Corbel, J. C. Andre and D. J. Lougnot, J. Polym. Sci. A - Polym. Chem. 30 (1992) 2715. 